\title{
X-RAY EMISSION FROM CATACLYSMIC VARIABLES
}

\author{
D. Q. Lamb \\ Department of Physics \\ Massachusetts Institute of Technology \\ and \\ Department of Physics \\ University of Illinois
}

Many cataclysmic variables have been found to be hard, as well as soft, $X$-ray sources. Emission from the boundary layer of an optically thick accretion disk extending down to the stellar surface can, at outburst, produce soft $x$-rays, but the production of hard $x$-rays from such a disk is difficult to understand. We therefore conjecture that the sources which emit hard $X$-rays have magnetic fields and are, in general, rotating. We then propose a classification scheme for cataclysmic variables based on the size of the Alfven radius $r$ relative to the stellar radius $R$ of the degenerate dwarf and the separation $a$ of the binary system. We show that many of the varied characteristics displayed by the cataclysmic variable $X$-ray sources can be understood in terms of this ordering. We suggest that the AM Her Class (AM Her, AN UMa, WV Pup, and 2A0311-23) have $R \ll a \ll r_{A}$, the $D Q$ Her Class (DQ Her, V533 Her, and $\mathrm{AE}$ Aqr) have $\mathrm{R} \ll r_{\mathrm{A}} \ll a$, while the SS Cyg Class (SS Cyg, U Gem, EX Hya, and GK Per) have $r_{A}{ }^{A} R \ll a$. Although $r_{A}$ depends on both the magnetic field strength of the degenerate dwarf and the accretion rate, for comparable rates of accretion the ordering that we propose is essentially one of decreasing magnetic field strength. 